\title{
Using Static Analysis to Reduce Dynamic Analysis Overhead*
}

\author{
Suan Hsi Yong (suan@cs.wisc.edu) \\ and Susan Horwitz (horwitz@cs.wisc.edu) \\ Computer Sciences Department, University of Wisconsin-Madison \\ 1210 West Dayton Street, Madison, WI 53706 USA
}

\begin{abstract}
Dynamic analysis (instrumenting programs with code to detect and prevent errors during program execution) can be an effective approach to debugging, as well as preventing harm from being caused by malicious code. One problem with this approach is the runtime overhead introduced by the instrumentation. We define several techniques that involve using the results of static analysis to identify some cases where instrumentation can safely be removed. While we have designed the techniques with a specific dynamic analysis in mind (that used by the Runtime Type-Checking tool), the ideas may be of more general applicability.
\end{abstract}

Keywords: Static Analysis, Dynamic Debugging, Runtime Types.

\section{Introduction}

Languages like $\mathrm{C}$ and $\mathrm{C}++$ that allow potentially unsafe operations such as pointer arithmetic, casting, and explicit memory management open the door to many difficult-to-detect errors. To identify such errors, a number of systems have been developed that involve dynamic analysis: instrumenting a program so that errors like out-of-bounds array indexes and bad pointer dereferences are detected when they occur during execution $[2,8,15,14,10,11]$. In some cases, dynamic checks are even mandated by the language definition (e.g., Java guarantees that an exception will be thrown whenever an array index is out of bounds, or a bad cast is performed).

Naturally, the benefits of dynamic analysis have an associated cost: the instrumentation introduces a certain amount of runtime overhead.

\footnotetext{
* This work was supported in part by the National Science Foundation under grants CCR-9970707 and CCR-9987435.
}

(C) 2003 Kluwer Academic Publishers. Printed in the Netherlands. 
This paper proposes several techniques for reducing the overhead by using static analysis to identify some cases in which instrumentation can safely be omitted. While the techniques were designed for one particular tool: the Runtime Type-Checking (RTC) tool [11], the ideas may be of more general applicability.

The remainder of the paper is organized as follows. Section 2 provides background on the RTC tool. Section 3 describes type-safetylevel analysis, a flow-insensitive analysis to identify "type-safe" expressions for which instrumentation can be eliminated. Section 4 presents redundant-check analysis, a flow-sensitive analysis to identify redundant instrumentation that can safely be removed. Section 5 presents experimental results showing the performance improvements gained from these analyses. Section 6 describes never-null-dereference analysis, another dataflow analysis to improve RTC performance. Section 7 discusses related work, and Section 8 concludes.

\section{The RTC Tool}

The RTC (Runtime Type-Checking) tool instruments C programs so that the runtime type of every memory location is tracked during program execution, and inconsistent type uses cause warnings and errors to be reported. Whenever a value $v$ is written into a location $l$, l's runtime type is updated with $v$ 's runtime type. Also, this runtime type is compared with l's declared type: if they do not match, a warning message is issued (a warning message is an indication that unusual behavior has been observed, and may be useful for diagnosing the root cause of a later error). Whenever the value in a location is used, its runtime type is checked, and if the type is inappropriate in the context in which the value is being used, an error message is issued; to avoid cascading error messages, the runtime type is set to the correct type after an error message is generated. 


\subsection{Motivating Examples}

While a number of other tools have been proposed to detect out-ofbounds array accesses and bad pointer dereferences, the type-checking approach of the RTC tool can also detect more subtle errors involving type misuses.

\section{EXAMPLE 1. Bad Union Access:}

A very simple example of a logical error that manifests itself as a bad runtime type is writing into one field of a union and then reading from another field with a different type. This is illustrated by the following code fragment:

$$
\begin{aligned}
& \text { 1. union } U\{\text { int } i \text {; float } f ;\} u \text {; } \\
& \text { 2. u.i }=10 ; \quad / * \text { write into u.i } * / \\
& \text { 3. u.f }=\text { u.f }+1.5 ; \quad / * \text { read from u.f } * /
\end{aligned}
$$

In this example, an integer value is written into variable $\mathrm{u}$ (on line 2 ), and is subsequently read and used as a float (on line 3). The RTC tool would track at runtime the type of the data stored at the single location corresponding to both $\mathrm{u} . \mathrm{i}$ and $\mathrm{u}$.f. That type would be set to int after the assignment $\mathrm{u} . \mathrm{i}=10$ on line 2 . On line 3 , when the value in that location is read and used as a float, the RTC tool would report a type mismatch error, because the runtime type associated with that location is int.

\section{EXAMPLE 2. Simulated Inheritence:}

Another class of subtle errors comes from a programming style in which $\mathrm{C}$ programmers simulate classes and inheritance using structures [18]. For example, the following declarations might be used to simulate the declaration of a superclass Base and a subclass Sub:

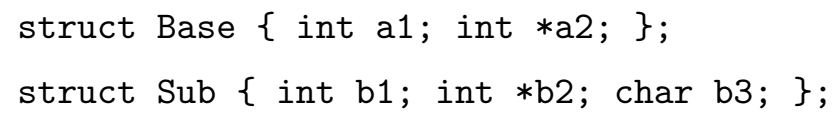

A function might be written to perform some operation on objects of the superclass: 


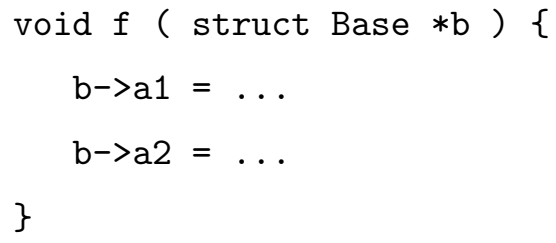

and the function might be called with actual arguments either of type struct Base * or struct Sub *:

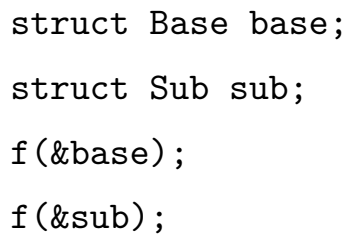

The ANSI C standard guarantees that the first field of every structure is stored at offset 0 , and that if two structures have a common initial sequence - an initial sequence of one or more fields with compatible types - then corresponding fields in that initial sequence are stored at the same offsets. Thus, in this example, fields a1 and b1 are both guaranteed to be at offset 0 , and fields a2 and b2 are both guaranteed to be at the same offset. Therefore, while the second call, $f$ (\&sub), would cause a compile-time warning (which could be averted with an appropriate type cast), it would cause neither a compile-time error nor a runtime error, and the assignments in function $f$ would correctly set the values of sub.b1 and sub.b2.

However, the programmer might forget the convention that struct Sub is supposed to be a subclass of struct Base, and while making changes to the code might change the type of one of the common fields, add a new field to struct Base without adding the same field to struct Sub, or add a new field to struct Sub before field b2. For example, suppose a new int field, i1 is added to struct Sub:

struct $\operatorname{Sub}\{$ int $b 1$; int $i 1$; int $* b 2$; char b3; \};

Now, when the second call to $f$ is executed, the assignment $b->a 2=\ldots$ would write into the $i 1$ field of sub rather than the b2 field. The fact that the b2 field is not correctly set by the call to $f$, or that the i1 field is overwritten with an unintended value, will probably either lead to a 
runtime error later in the execution, or cause the program to produce incorrect output.

The tracking of runtime types performed by the RTC tool can help the programmer uncover the source of this logical error. The assignment $\mathrm{b}->\mathrm{a} 2=\ldots$ causes sub.i1 to be tagged with type pointer. A later use of sub.i1 in a context that requires an int would result in an error message due to the mismatch between the required type (int) and the current runtime type (pointer).

Note that in this example, a tool like Purify [8] would not report any errors, because there are no bad pointer or array accesses: function $f$ is not writing outside the bounds of its structure parameter, it just happens to be the wrong part of that structure from the programmer's point of view.

\subsection{Tracking Types}

The RTC tool associates with each memory location one of the following runtime types: unallocated, uninitialized, pointer, zero, char, short, int, long, float, double. The first two are used to tag unallocated and uninitialized memory respectively; pointers to different types are tagged with the same runtime type pointer, and so are treated as compatible with each other; the special zero type is used to tag memory locations that are assigned the literal 0 , and is treated as being compatible with all other types. The remaining types correspond to $\mathrm{C}$ scalar types. ${ }^{1}$ For aggregate objects (structures and arrays), the runtime type of each field/element is tracked separately; typedefs are resolved to their underlying basic type. The runtime types are stored in a "mirror" of the memory used by the program, with each byte of memory mapped to a four-bit nibble in the mirror (thus incurring a $50 \%$ space overhead).

The RTC tool has been implemented to handle all of ANSI C. Using Ckit[5] as its front end, it translates a given set of preprocessed $\mathrm{C}$ source

\footnotetext{
1 The RTC tool actually represents $\mathrm{C}$ scalar types using a pair $\langle\sigma$, size $\rangle$, where $\sigma$ is one of $\{$ integral, real $\}$, and size is the size (in bytes) of the type. For example, on an x86 Linux, a char would be represented by $\langle$ integral, 1$\rangle$, and a float by $\langle$ real, 4$\rangle$.

This means, in fact, that int and long are not differentiated, and also that larger types like long long can also be represented. In this paper, these subtleties are ignored for simplicity.
} 
files into instrumented $\mathrm{C}$ files. These are then compiled and linked with the RTC library, producing an executable that performs runtime type checking and reports error and warning messages.

The instrumentation phase is a source-to-source translation of the C program; it performs a syntax-directed transformation on the program's abstract-syntax tree to add calls to RTC library functions that track the runtime types. The following operations are performed by these library functions:

declare - a variable declaration is instrumented to set the runtime type in the variable's mirror to uninitialized. (Initially, the mirror for all memory is implicitly tagged unallocated.)

clear - when a variable is deallocated (at function return for stack variables, and during free for heap locations), the mirror for that location is tagged unallocated.

copy - an assignment statement is instrumented to copy the runtime type of the right-hand-side value into the mirror of the left-hand-side location; additionally, if the runtime type of the assigned value does not match the static type of the assignment, a warning message is issued.

verify - a use of a memory location $x$ in the context of a type $\tau$ is instrumented to compare the runtime type in the mirror of $x$ with $\tau$. If the types are not compatible, an error message is issued, and the runtime type of $x$ is corrected to $\tau$ (to prevent cascading error messages).

verify-pointer - a pointer dereference is instrumented to check whether the mirror of the pointer's target is unallocated. If it is, an error message is issued. This check detects dangling pointer dereferences, dereferences of certain stray pointers (those that point between or beyond allocated blocks), and also null-pointer dereferences (because the mirror of memory location 0 is tagged unallocated).

The tool is designed so that instrumented modules can be linked with uninstrumented ones. This flexibility is useful if, for example, a programmer only wants to debug one small component of a large program: they can instrument only the files of interest, and link them 
with the remaining uninstrumented object modules. A caveat when doing this, however, is that it may lead to spurious warning and error messages because the uninstrumented parts of the code do not maintain the necessary runtime type information for the memory locations they declare or use. For example, if a reference to a valid object declared in the uninstrumented portion of the program is passed to an instrumented function, the tool will consider that object unallocated, and may output a spurious error message if that object is referenced.

This problem extends, in general, to library modules. For example, the runtime types associated with the flow of values in a function like memcpy, the initialization of values from input in a function like fgets, and the types of the data in a static buffer returned by a function like ctime would not be captured. To handle these, we have created a collection of instrumented versions of common library functions that affect type flow. These are wrappers of the original functions, handwritten to perform the necessary tag-update operations in the RTC mirror to capture their type behavior.

Included among these instrumented library functions are memorymanagement functions. Each call to malloc (or one of its relatives) is replaced with a call to a wrapper version which, upon successfully allocating a block of memory, sets the mirror for that memory block to uninitialized (or zero for calloc). Similarly, the wrapper version of the free function resets the mirror to unallocated. The malloc wrapper also adds padding between allocated blocks to decrease the likelihood of a stray pointer jumping from one block to another (this is the approach used by Purify [8]).

The RTC tool was able to detect bugs in some SPEC 95 benchmarks (go, ijpeg), Solaris utilities (nroff, col, etc.), and Olden benchmarks (health, voronoi) [11]. Most of the errors were out-of-bounds array or pointer accesses. In the Solaris utilities, the out-of-bounds accesses resulted in program crashes; in the SPEC cases, the errors had no apparent effect on the execution, which made the errors difficult to detect without the use of a tool like RTC. In every case, the RTC tool was able to detect the out-of-bounds memory accesses because the type associated with the pointed-to memory was different from the expected type. 
Finally, the RTC tool lends itself naturally to interactive debugging. When a warning or error message is issued, a signal (SIGUSR1) is sent, and can be intercepted by an interactive debugger like GDB [19]. The user can then examine memory locations, including the mirror, and make use of GDB's features to help track down the cause of an error.

A major shortcoming of the RTC tool is poor performance: in the worst case, an instrumented program ran 130 times slower than the noninstrumented version. This is because the RTC tool instruments every expression in the program and tracks the runtime type of every memory location used in the program. To reduce the overhead of the RTC instrumentation, we implemented two static analyses to identify and remove unnecessary instrumentation: type-safety-level analysis, and redundant checks analysis.

\section{Type-Safety-Level Analysis}

Our first static analysis is a flow-insensitive type-safety-level analysis that partitions the expressions in a program into levels of "type safety", so that certain classes of runtime instrumentation can be eliminated for expressions at certain type-safety levels.

For this description, we assume that the assignment statements in the input program have been normalized to the forms defined by the following context-free grammar:

$$
\begin{aligned}
& \text { assign } \Rightarrow \text { lvalue }=\text { rvalue } \\
& \mid \text { lvalue }=(\tau)_{\text {cvt }} \text { rvalue } \\
& \mid \quad \text { lvalue }=(\tau)_{\text {ext }} \text { rvalue } \\
& \mid \quad \text { lvalue }=(\tau)_{\text {cpy }} \text { rvalue } \\
& \text { lvalue } \Rightarrow \text { var } \mid * \text { var } \\
& \text { rvalue } \Rightarrow \text { const } \mid \text { var } \mid * \text { var } \mid \text { \&var } \mid \text { var } \oplus \text { var }
\end{aligned}
$$

where const is a constant, var is a variable, and $\oplus$ represents any $\mathrm{C}$ binary operator. This simplified language captures the essence of $\mathrm{C}$ assignment statements; details of how to handle other $\mathrm{C}$ constructs

(e.g., structures, unions, and function calls) are omitted for brevity. 
An assignment that involves an array index, such as $x=a[i]$, can be rewritten as tmp $=a+i ; x=* t m p$.

Typecasts are divided into three forms. The first form, $(\tau)_{c v t} e$, is a typecast that involves a change in representation, and includes conversions (e.g., between integers and floating-point values) and truncation of data (e.g., when type-casting a long int into a short int). The second form, $(\tau)_{\text {ext }} e$, represents type-casts that extend data from a smaller type to a larger type with no change in the data bits (e.g., from a short int into a long int). The third form, $(\tau)_{c p y}$, represents typecasts where there is no change in the form of the data, and includes casts between pointers and integers (of the same size). The difference between these forms that concerns us is that a conversion cast, $(\tau)_{c v t} e$, is treated by the RTC tool as a use of $e$ that always returns a value of type $\tau$, regardless of whether $e$ 's runtime type is compatible with its static type (if it is not, the RTC instrumentation issues an error message, then sets $e$ 's runtime type to its static type to avoid cascading error messages). Extension and copy casts $(\tau)_{\text {ext }} e$ and $(\tau)_{\text {cpy }} e$ are treated as bitwise copies, whose runtime type is the same as the runtime type of $e$.

\subsection{Points-to Analysis}

The type-safety-level analysis described below uses points-to analysis to account for possible aliasing in the program. Points-to analysis associates each pointer $p$ with a points-to set, which is the set of variables that $p$ may point to during program execution. For our implementation, we used Das's flow-insensitive points-to analysis [6], which is scalable and has good precision. Other (flow-insensitive) points-to analyses can also be used (e.g., [1, 22]). In general, better precision in the points-to analysis would enable the elimination of a greater amount of unnecessary instrumentation, thus potentially leading to greater improvement in the performance of the RTC-instrumented program. 


\subsection{TyPE-SAFEty LeVELS}

The main idea behind the analysis is to classify all lvalue expressions ${ }^{2}$ in the program into the following type-safety levels:

safe - An expression whose runtime type is guaranteed always to be compatible with its static type, and for which all instrumentation can be eliminated (assuming there are no uses of uninitialized values; Section 3.6 describes how we identify locations that may be uninitialized).

unsafe - An expression whose runtime type may be incompatible with its static type. This includes expressions of the form *p, when the pointer $\mathrm{p}$ may be NULL, or may contain an invalid address. An unsafe expression must be fully instrumented.

tracked - A location whose runtime type is always compatible with its static type, but which may be pointed to by a pointer $p$ such that $* p$ is unsafe. A tracked location needs to have its runtime type initialized (to its static type) in the mirror, but instrumentation for verifying and copying its runtime type can be eliminated.

Figure 1 (a) presents an example code fragment to illustrate the intuition behind the approach. Since the approach is flow-insensitive, the order of the statements is ignored in the analysis.

Figure 1 (b) gives the type-safety levels we wish to identify for the expressions in the example program. The expressions p0, p1 and p2 are safe because they are only assigned pointer-typed values (recall that the RTC tool does not differentiate between pointer types, so the fact that $\mathrm{p} 1$ is assigned both the address of an int variable and the address of a float variable is not important; also recall that the literal 0 is treated as being compatible with all types, including pointers).

The expressions $\mathrm{f}, * \mathrm{p} 0, * \mathrm{p} 1$, and $* \mathrm{p} 2$ are all unsafe. Variable $\mathrm{f}$ is unsafe because the assignment at line 13 could write an int value into

\footnotetext{
${ }^{2}$ An expression that represents a location in memory is called an lvalue expression. In the simplified language described in this paper, these are either of the form var or *var. In the full $\mathrm{C}$ version we have implemented, lvalue expressions also include expressions like structure members (var.id) and multi-level dereferences $(* * \operatorname{var})$.
} 


\begin{tabular}{|c|c|c|}
\hline (a) & Code & (b) Type-safety levels \\
\hline 1. & int $i$; & safe: $\mathrm{p} 0, \mathrm{p} 1, \mathrm{p} 2$ \\
\hline 2 . & int $* \mathrm{p} 0, * \mathrm{p} 1, * \mathrm{p} 2$ & unsafe: $\mathrm{f}, * \mathrm{p} 0, * \mathrm{p} 1, * \mathrm{p} 2$ \\
\hline 3. & float $f$; & tracked: $\mathrm{i}$ \\
\hline & & (c) Assignment Edges \\
\hline 4. & $i=1$ & $i \stackrel{\text { VALUE }_{i n t}}{\longleftarrow}$ \\
\hline 5 . & $f=2.3$ & $f \stackrel{=}{\longleftarrow} \operatorname{VALUE}_{\text {float }}$ \\
\hline 6. & $\mathrm{p} 0=0$ & $p 0 \stackrel{=}{\longleftarrow} \operatorname{VALUE}_{z e r o}$ \\
\hline 7 . & if $(* \mathrm{p} 0==0)$ & \\
\hline 8 . & $\mathrm{p} 1=\& i$ & $p 1 \stackrel{=}{=}$ VALUE $_{\text {valid }-p t r}$ \\
\hline 9. & else & \\
\hline 10. & $\mathrm{p} 1=($ int $*) \& f$ & $p 1 \stackrel{=}{\longleftarrow}$ VALUE $_{\text {valid-ptr }}$ \\
\hline 11. & $\mathrm{p} 2=\mathrm{p} 1+i$ & $p 2 \stackrel{=}{\longleftarrow} \oplus_{p t r} p 1 \quad p 2 \stackrel{=}{\longleftarrow} \oplus_{p t r} i$ \\
\hline 12. & if $(* p 2 \quad !=0)$ & \\
\hline 13. & $* \mathrm{p} 1=4 ;$ & $* p 1 \stackrel{=}{=}$ VALUE $_{i n t}$ \\
\hline
\end{tabular}

Figure 1. Type-safety example.

f via $* \mathrm{p} 1$. The expression $* \mathrm{p} 0$ is unsafe because $\mathrm{p} 0$ may be NULL (due to the assignment at line 6$) ; * \mathrm{p} 1$ and $* \mathrm{p} 2$ are unsafe because while they each have a static type of $i n t, * \mathrm{p} 1$ may refer to a float (because of the assignment at line 10 ), and $* \mathrm{p} 2$ may refer to an invalid address (as a result of the pointer arithmetic at line 11).

Finally, the expression $i$ is tracked. Although $i$ will always contain an int value, it may be pointed to by $\mathrm{p} 1$, and $* \mathrm{p} 1$ is unsafe. This means that every use of $* \mathrm{p} 1$ will be instrumented to check its runtime type, and so every location in p1's points-to set — including i - must have its runtime type tracked in the mirror.

Within this framework, we can devise schemes of varying precision to determine the type-safety level of each expression. Using the following ordering,

$$
\text { unsafe }<\text { tracked }<\text { safe }
$$

any scheme that classifies each expression at a level less than or equal to its true level is a safe approximation. For example, the unoptimized RTC tool corresponds to one extreme, where all expressions are 
considered unsafe. The next three sections describe an efficient flowinsensitive analysis to classify the type-safety level of expressions. The analysis works as follows:

Step 1: Build an assignment graph in which the nodes represent the expressions in the program, and the edges represent the flow of runtime types due to assignments.

Step 2: Compute a runtime-type attribute, rt-type, for each node in the graph.

Step 3: Compute the type-safety level for each lvalue node in the graph.

\subsection{Step 1: Building the Assignment Graph}

The first step of the analysis involves building an assignment graph that records the flow of runtime types among the expressions in the program. Each node in the assignment graph corresponds to an expression, and represents an "abstract object" of one of four forms: $v, * v, \oplus_{\tau} v$, and $\operatorname{VALUE}_{\tau}$. The $v$ node represents a variable $v ; * v$ represents a dereference; $\oplus_{\tau} v$ represents an arithmetic, logical, or bitwise operation on $v$ (resulting in a value of static type $\tau$ ); and $\operatorname{VALUE}_{\tau}$ represents a value of type $\tau$, e.g., from a constant expression. For both $\oplus_{\tau} v$, and $\operatorname{VALUE}_{\tau}$, $\tau$ will be either a scalar C type - char, int, float, etc. - or one of the following special types:

valid-ptr: A pointer expression that is guaranteed to evaluate to a valid address (the address of an allocated memory location) has type valid-ptr. For example, the expression \& $\mathrm{x}$ has type valid-ptr.

pointer: A pointer expression that may evaluate to an invalid address (including NULL) has type pointer. For example, the expression $\& \mathrm{x}+\mathrm{k}$ has type pointer.

zero: An expression that is guaranteed to evaluate to 0 has type zero. For example, the literal 0 has type zero. 


\begin{tabular}{ccl}
\hline expr & AbsObj(expr $)$ & Notes \\
\hline 0 & $\left\{\right.$ VALUE $\left._{z e r o}\right\}$ & \\
$C_{\tau}$ & $\left\{\right.$ VALUE $\left._{\tau}\right\}$ & $C_{\tau}$ is a non-zero constant of type $\tau$ \\
$\& y$ & $\left\{\right.$ VALUE $\left._{\text {valid-ptr }}\right\}$ & \\
$y$ & $\{y\}$ & \\
$* y$ & $\{* y\}$ & \\
$y \oplus z$ & $\left\{\oplus_{\tau} y, \oplus_{\tau} z\right\}$ & The expression $y \oplus z$ has static type $\tau$ \\
\hline
\end{tabular}

(a)

\begin{tabular}{|c|c|}
\hline Assignment & Edge(s) in Graph \\
\hline$e_{1}=e_{2}$ & $n_{1} \stackrel{=}{=} n_{2}, n_{1} \in \operatorname{AbsObj}\left(e_{1}\right), n_{2} \in \operatorname{Abs} \operatorname{Obj}\left(e_{2}\right)$ \\
\hline$e_{1}=(\tau)_{c p y} e_{2}$ & $n_{1} \stackrel{=}{=} n_{2}, n_{1} \in \operatorname{AbsObj}\left(e_{1}\right), n_{2} \in \operatorname{Abs} \operatorname{Obj}\left(e_{2}\right)$ \\
\hline$e_{1}=(\tau)_{c v t} e_{2}$ & $n_{1} \stackrel{c v t}{\longleftarrow} n_{2}, n_{1} \in \operatorname{AbsObj}\left(e_{1}\right), n_{2} \in \operatorname{AbsObj}\left(e_{2}\right)$ \\
\hline$e_{1}=(\tau)_{e x t} e_{2}$ & $n_{1} \stackrel{e x t}{\longleftarrow} n_{2}, n_{1} \in \operatorname{AbsObj}\left(e_{1}\right), n_{2} \in \operatorname{AbsObj}\left(e_{2}\right)$ \\
\hline
\end{tabular}

(b)

Figure 2. Rules for initializing the assignment graph.

Nodes are connected by three kinds of (directed) assignment edges: conversion edges $(\stackrel{\text { cvt }}{\longrightarrow})$, extension edges $(\stackrel{\text { ext }}{\longrightarrow})$, and copy edges $(\stackrel{\longrightarrow}{\longrightarrow})$. Conversion edges represent assignments with a right-hand side of the form $(\tau)_{c v t} e$, extension edges represent assignments with a right-hand side of the form $(\tau)_{e x t} e$, and copy edges represent assignments that do not involve a type-cast, or that involve a type-cast of the form $(\tau)_{c p y} e$.

Figure 2(a) shows the mapping $A b s O b j$ from program expressions to abstract objects, while Figure 2(b) gives the rules for adding edges to the graph. For example, the assignment $x=y \oplus z$ adds to the graph two copy edges, $x \stackrel{=}{=} \oplus_{\tau} y$ and $x \stackrel{=}{=} \oplus_{\tau} z$, where $\tau$ is the static type of the expression $y \oplus z$.

Figure 1 (c) gives the assignment edges derived from the the example program. 


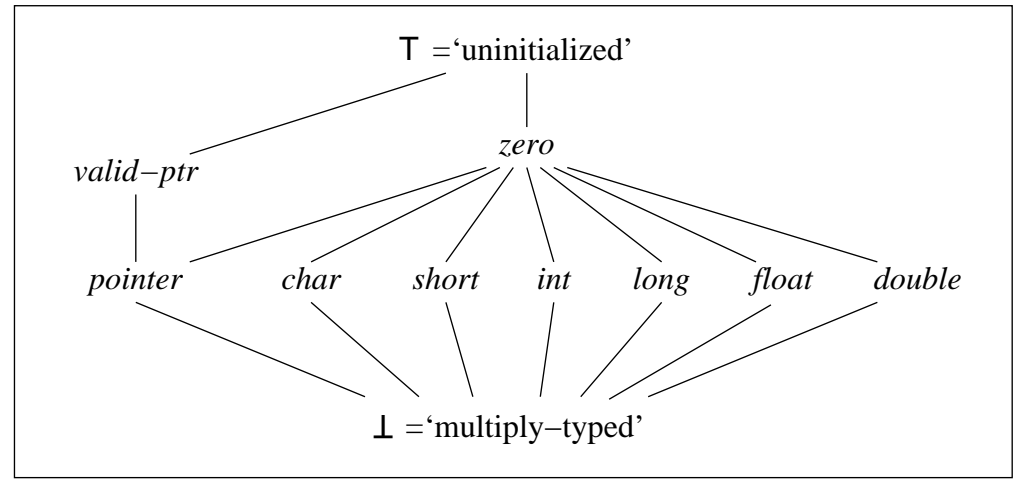

Figure 3. The lattice for rt-type.

\subsection{Step 2: Computing Runtime Types}

After building the assignment graph, the analysis computes a runtimetype attribute, $r$-type $(n)$, for each node $n$ in the graph. The values of rt-type form the lattice shown in Figure 3. Intuitively, rt-type $(n)$ summarizes the set of types that the expression corresponding to $n$ might have at runtime. A rt-type of $\perp$ means that an expression could have more than one incompatible runtime type.

Figure 4 gives the constraints for computing rt-type for each node in the assignment graph. In the figure, $p t-s e t(p)$ is the points-to set of $p$, while static-type $(n)$ is the static type of the expression represented by $n$.

Rules T1 and T2 set the $r$-type of each $\mathrm{VALUE}_{\tau}$ node and each $\oplus_{\tau} x$ node to be its static type $(\tau)$. For a $\oplus_{\tau} x$ node, the $r$-type is $\tau$ because the value of an expression such as $\mathrm{x}+\mathrm{y}$ is considered by the RTC to have a runtime type equal to the static type of the expression. Rule T3 constrains the rt-type of a $* p$ node to be no higher in the lattice than the type of each variable in $p$ 's points-to set. Rule T4 constrains the rt-type of the left-hand side of a conversion to be no higher than its static type; this is because the RTC tool always treats a conversion cast as having a runtime type equal to its static type. Rule T5 deals 


\begin{tabular}{|c|c|c|}
\hline & Condition & Inferred Constraint \\
\hline T1. & & $r t$-type $\left(\operatorname{VALUE}_{\tau}\right)=\tau$ \\
\hline $\mathrm{T} 2$. & & $r$-type $\left(\oplus_{\tau} x\right)=\tau$ \\
\hline T3. & $x \in p t-\operatorname{set}(p)$ & $r t$-type $(* p) \sqsubseteq r t$-type $(x)$ \\
\hline $\mathrm{T} 4$. & $n_{1} \stackrel{c v t}{\longleftarrow} n_{2}$ & $r t$-type $\left(n_{1}\right) \sqsubseteq$ static-type $\left(n_{1}\right)$ \\
\hline T5. & $n_{1} \stackrel{e x t}{\longleftarrow} n_{2}$ & $\begin{array}{l}\text { if } r t \text {-type }\left(n_{2}\right)==\text { static-type }\left(n_{2}\right) \\
\text { then } \quad \text { rt-type }\left(n_{1}\right) \sqsubseteq \text { static-type }\left(n_{1}\right) \\
\text { else } \quad r t \text {-type }\left(n_{1}\right)=\perp\end{array}$ \\
\hline T6a. & $\begin{array}{l}* p \stackrel{=}{=} n_{2} \\
x \in p t-\operatorname{set}(p)\end{array}$ & $r t$-type $(x) \sqsubseteq r t$-type $\left(n_{2}\right)$ \\
\hline T6b. & $\begin{array}{l}n_{1} \stackrel{=}{=} n_{2} \\
n_{1} \text { not of } \\
\text { the form } * p\end{array}$ & $r t$-type $\left(n_{1}\right) \sqsubseteq r t$-type $\left(n_{2}\right)$ \\
\hline
\end{tabular}

Figure 4. Rules for computing rt-type.

with extension edges: if the right-hand side of an extension assignment is well-typed, then the rt-type of the left-hand side is constrained to be no higher than its static type; otherwise, since the assignment will effectively be copying an aggregation of multiple RTC tags into the left-hand side, the rt-type of the left-hand side is set to $\perp$. Rules T6a and T6b handle assignment edges: if the left-hand side is a dereference of a pointer $p$ (rule T6a), then the rt-type of each node in the points-to set of $p$ can be no higher than the rt-type of the right-hand side; if the left-hand side is a variable $v$ (rule T6b), then its rt-type can be no higher than the rt-type of the right-hand side.

The rt-type values are computed for the nodes in the assignment graph by solving the constraints generated from the above rules. This is done by building a directed graph with the same nodes as the assignment graph, and edges representing $\sqsubseteq$ constraints induced by rules T3, T6a, and T6b (and collapsing cycles for efficiency). Initially, all nodes are given $r$-type $=T$. Next, rules T1, T2, T4, and T5 are used to assign rt-type values to the nodes in the graph where these rules apply (rules T4 and T5 are used to assign static-type $\left(n_{1}\right)$ to the node that represents $n_{1}$ ). The graph is then traversed to propagate rt-type values along the $\sqsubseteq$ edges: for each node $n$, rt-type $(n)$ is assigned the meet of the old value of rt-type( $n)$ with the rt-type of all $n^{\prime}$ for which there is a constraint edge representing $n \sqsubseteq n^{\prime}$. After propagation, we 


\begin{tabular}{|c|c|c|}
\hline Assignments & Assignment Edges & Inferred Constraints \\
\hline \multirow{7}{*}{$\begin{array}{l}i=1 ; \\
f=2.3 ; \\
p 0=0 ; \\
p 1=\& i ; \\
p 1=(i n t *) \& f ; \\
\text { p2 }=p 1+i ;\end{array}$} & $i \stackrel{=}{=}$ VALUE $_{i n t}$ & $r t$-type $(i) \sqsubseteq i n t$ \\
\hline & $f \stackrel{\text { VALUE }_{\text {float }}}{=}$ & rt-type $(f) \sqsubseteq$ float \\
\hline & $p 0 \stackrel{=}{=} \operatorname{VALUE}_{z e r o}$ & $r t$-type $(p 0) \sqsubseteq z e r o$ \\
\hline & $p 1 \stackrel{=\text { VALUE }_{\text {valid }-p t r}}{ }$ & $r$-type $(p 1) \sqsubseteq$ valid-ptr \\
\hline & $p 1 \stackrel{=}{=}$ VALUE $_{\text {valid }-p t r}$ & $r t$-type $(p 1) \sqsubseteq$ valid-ptr \\
\hline & $\begin{array}{l}p 2 \stackrel{=}{=} \oplus_{\text {pointer }} p 1 \\
p 2 \stackrel{=}{=} \oplus_{\text {pointer }} i\end{array}$ & $\begin{array}{l}\text { rt-type }(p 2) \sqsubseteq \text { pointer } \\
\text { rt-type }(p 2) \sqsubseteq \text { pointer }\end{array}$ \\
\hline & $\begin{array}{l}* p 1 \stackrel{=}{\longleftarrow} \text { VALUE }_{i n t} \\
(p t-\operatorname{set}(p 1)=\{i, f\})\end{array}$ & $\begin{array}{l}r \text {-type }(i) \sqsubseteq \text { int } \\
r t \text {-type }(f) \sqsubseteq i n t \\
r t \text {-type }(* p 1) \sqsubseteq r t \text {-type }(i) \\
r t \text {-type }(* p 1) \sqsubseteq r t \text {-type }(f)\end{array}$ \\
\hline
\end{tabular}

\begin{tabular}{|ll|}
\hline Final $r$-type values: & \\
\hline$r$-type $(p 0)=$ zero & $r$-type $(i)=i n t$ \\
$r t$-type $(p 1)=$ valid-ptr & $r$-type $(f)=\perp$ \\
$r t$-type $(p 2)=$ pointer & $r t$-type $(* p 1)=\perp$ \\
\hline
\end{tabular}

Figure 5. Computing rt-type for the example in Figure 1.

must revisit all extension edges (in the original assignment graph), and check against rule T5: if $r$-type $\left(n_{2}\right) \neq$ static-type $\left(n_{2}\right)$, and $r$-type $\left(n_{1}\right)$ is not already $\perp$, then $r$-type $\left(n_{1}\right)$ is set to $\perp$, and the graph must be traversed again to propagate this change. Since extension casts are rare in practice, this does not noticeably affect efficiency.

Figure 5 shows the assignment edges, inferred constraints, and final rt-type values for the example of Figure 1.

\subsection{Step 3: Computing Type-Safety Levels}

Once the rt-type values are computed, each node of the graph is annotated with an attribute signifying its type-safety level - either unsafe or tracked - based on the rules given in Figure 6; after applying the rules, any node not annotated unsafe or tracked is considered safe.

Rule L1 annotates as unsafe any node whose rt-type is not compatible with its static-type (recall that in the lattice for rt-type, valid-ptr is compatible with pointer, and zero is compatible with all scalar types). 


\begin{tabular}{|c|c|c|}
\hline & Condition & Attribute \\
\hline L1. & rt-type $(n) \nsupseteq$ static-type $(n)$ & $n$ unsafe \\
\hline $\mathrm{L} 2$. & $\begin{array}{l}\text { static-type }(p)==\text { pointer } \\
\text { rt-type }(p) \neq \text { valid-ptr }\end{array}$ & $* p$ unsafe \\
\hline L3. & $\begin{array}{l}* p \text { unsafe } \\
x \in \text { pt-set }(p) \\
x \neq \text { unsafe }\end{array}$ & $x$ tracked \\
\hline
\end{tabular}

Figure 6. Rules for determining type-safety levels.

For Rule L2: a pointer $p$ whose $r$-type is not valid-ptr may contain an invalid address, therefore $* p$ must be instrumented to check its runtime type, and is thus annotated as unsafe. Rule L3 annotates as tracked any variable in the points-to set of a pointer $p$ whose dereference node $(* p)$ is unsafe.

Looking back at the example in Figure 5, Rule L1 makes $f$ and $* p 1$ unsafe, L2 makes $* p 0$ and $* p 2$ unsafe, and L3 makes $i$ tracked. This leaves $p 0, p 1$ and $p 2$ as safe.

Note that since safe variables are not instrumented, a safe variable $v$ will always be tagged unallocated in the mirror. If the contents of $v$ is accessed indirectly by an errant pointer $p$, the dereference expression $* p$ will have been annotated as unsafe, and thus will be instrumented with a verify-pointer operation. Since $v$ is tagged unallocated, the check of $* p$ will trigger an "accessing unallocated memory" error. Thus, by identifying safe variables, the type-safety-level analysis not only lowers the overhead of the tool by eliminating unnecessary instrumentation, it also increases the likelihood of the RTC tool detecting an error, because it effectively tags more memory as unallocated.

\subsection{MAY-BE-Uninitialized AnAlysis}

The type-safety-level analysis described above does not account for uses of uninitialized data, which is an error that the RTC tool checks for. That is, by eliminating all instrumentation for safe and tracked locations, and by initializing tracked locations to their static types rather than to uninitialized, the RTC tool will no longer detect uses 
of uninitialized data in these locations. To address this problem, an additional flow-sensitive analysis is needed to find program points where instrumentation cannot be elided.

For a location $x$ that is safe or tracked, the analysis finds instances of $x$ where $x$ may be uninitialized. This analysis is defined as a dataflowanalysis problem on a control-flow graph (CFG):

- The elements of the underlying lattice are sets of locations (variables or abstract locations representing heap objects).

- The analysis computes two sets for each CFG node $n$ : Uninitin $(n)$ and $\operatorname{Uninit}_{\text {out }}(n)$, representing locations that may be uninitialized before and after $n$.

- The lattice meet is set union.

The dataflow transfer functions reflect how the RTC tool treats locations tagged as uninitialized. If $y$ is uninitialized, an assignment $x=y$ is instrumented to copy the uninitialized tag into the mirror for $x$. But for a use of $y$, such as $x=y+1$, if $y$ is uninitialized, the RTC verify function will report an error, and set $x$ 's runtime type to its static type (to avoid cascading errors).

The dataflow transfer functions are as follows:

- At a node $n$ that declares the variable $x$, or allocates the heap location $x$ (e.g., via a call to malloc), Uninit ${ }_{\text {out }}(n)=\operatorname{Uninit}_{i n}(n) \cup$ $\{x\}$

- At a node $n$ that is a direct assignment $x=e$ (where $x$ is a variable),

- if $e$ is a variable $y$ such that $y \in \operatorname{Uninitin}_{i n}(n)$, then Uninit $_{\text {out }}(n)=\operatorname{Uninit}_{\text {in }}(n) \cup\{x\}$

- if $e$ is a dereference $* q$ such that $p t-\operatorname{set}(q) \cap \operatorname{Uninit}_{i n}(n) \neq \emptyset$, then $\operatorname{Uninit}_{\text {out }}(n)=$ Uninit $_{\text {in }}(n) \cup\{x\}$

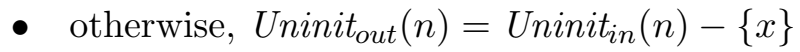

- At a node $n$ that is an indirect assignment $* p=e$, 
- if $e$ is a variable $y$ such that $y \in \operatorname{Uninitin}_{i n}(n)$, then $\operatorname{Uninit}_{\text {out }}(n)=\operatorname{Uninit}_{\text {in }}(n) \cup p t-\operatorname{set}(p)$

- $\quad$ if $e$ is a dereference $* q$ such that $p t$-set $(q) \cap \operatorname{Uninit}_{i n}(n) \neq \emptyset$, then $\operatorname{Uninit}_{\text {out }}(n)=\operatorname{Uninit}_{\text {in }}(n) \cup$ pt-set $(p)$

- $\quad$ otherwise, $\operatorname{Uninit}_{\text {out }}(n)=\operatorname{Uninit}_{i n}(n)$

To account for function calls, which is an important aspect to consider in dataflow analysis, we augment the language used in our description to include simple function calls, $f()$, with no arguments or return values (arguments and return values can be modeled by assignments to globals). The dataflow transfer function for a function call is:

- At a node $n$ containing a call $\mathrm{f}()$,

$$
\operatorname{Uninit}_{\text {out }}(n)=\operatorname{Uninit}_{\text {in }}(n) \cup \operatorname{MayMod}(\mathbf{f})
$$

where $\operatorname{MayMod}(\mathrm{f})$ is the set of locations that may be modified as a result of calling the function. Essentially, a call to $f$ is treated as possibly assigning an uninitialized value to all locations in $\operatorname{MayMod}(\mathbf{f})$.

After performing the may-be-uninitialized analysis, instrumentation is added to allow the RTC tool to detect uses of uninitialized data. A tracked or safe location $x$ for which there is a use of $x$ in a node $n$ where $x \in \operatorname{Uninit}_{\text {in }}(n)$ is treated as follows:

1. The declaration of $x$ is instrumented with a declare operation that sets its type in the mirror to uninitialized.

2. For each node $n$ that uses $x$, if $x \in \operatorname{Uninitin}_{\text {in }}(n)$, then the use of $x$ is instrumented with a verify operation.

3. For each node $n$ that defines $x$, if either $x \in \operatorname{Uninit}_{i n}(n)$ or $x \in \operatorname{Uninit}_{\text {out }}(n)$, then the assignment is instrumented with a copy operation (this ensures that $x$ 's tag is set correctly for subsequent uses of $x$ ). The only definitions of $x$ that need not be instrumented are those for which $x$ is definitely not uninitialized both before and after the definition. 


\section{Redundant-check Analysis}

When a location $x$ is read many times with no intervening writes, a runtime check is only necessary for the first read of $x$. This is because if the first check of $x$ reports a runtime error, the RTC tool will set its runtime type to its (statically) declared type to prevent cascading errors.

A subsequent check of the same location, with no intervening writes to that location, is therefore redundant, and should be eliminated.

To identify redundant checks, we perform a dataflow analysis to keep track of lvalue expressions that have been checked. A check of an unsafe expression $e$ is redundant at control-flow graph node $n$ if every path from the CFG's enter node to $n$ includes a node $m$ such that:

- There is a use of $e$ at node $m$ (where $e$ will be instrumented by the RTC tool to verify its runtime type), and

- No path from $m$ to $n$ changes the runtime type of $e$, changes the l-value of $e$, or deallocates location $e$.

The analysis is defined as a Gen/Kill dataflow problem:

- The elements of the underlying lattice are sets of unsafe lvalue expressions (either a variable $v$ or a pointer dereference $* p$ ).

- The analysis computes two sets for each CFG node $n$ : Checked $_{i n}(n)$ and Checked out $_{(}(n)$, representing expressions for which checks are redundant before and after $n$.

- The dataflow transfer function for each node $n$ is of the form Checked $_{\text {out }}(n)=$ Checked $_{\text {in }}(n)-\operatorname{Kill}_{(n)} \cup \operatorname{Gen}(n)$

- The lattice meet is set intersection.

The set $\operatorname{Gen}(n)$ for a node $n$ includes the lvalue expression $e$ if $e$ is unsafe and there is a use of $e$ at node $n$; that is, $e$ will be instrumented to verify its runtime type. The set $\operatorname{Kill}(n)$ for a node $n$ is described by the conditions listed below.

A variable $v$ is in $\operatorname{Kill}(n)$ for a node $n$ containing any of the following: 
1. An assignment $v=e$, where $e$ is an unsafe expression.

2. An assignment $* p=e$, where $e$ is an unsafe expression, and $v$ is in the points-to set of $p$.

3. A call $\mathrm{f}()$, where $v$ is in $\operatorname{MayMod}(\mathbf{f})$.

A dereference expression $* p$ is in $\operatorname{Kill}(n)$ for a node $n$ containing any of the following:

4. An assignment $x=e$, where $e$ is an unsafe expression, and $x$ is in the points-to set of $p$.

5. An assignment $* q=e$, where $e$ is an unsafe expression, and the points-to sets of $p$ and $q$ intersect.

6. Any assignment to $p$.

7. Any assignment to $* q$, where $p$ is in the points-to set of $q$.

8. A call $\mathrm{f}()$, where $\operatorname{MayMod}(\mathrm{f}) \cap p t-\operatorname{set}(p) \neq \emptyset$.

9. A call $\mathrm{free}(q)$, where the points-to sets of $p$ and $q$ intersect.

Note that r-value expressions of the form const, \&x, or $e_{1} \oplus e_{2}$ always evaluate to well-typed values, and are thus considered safe expressions. For condition 9 , we treat calls to free differently from other function calls, to account for memory deallocation.

After performing this analysis, a use of an expression $e$ at a node $n$ need not be instrumented if $e$ is in $\operatorname{Checked}_{i n}(n)$

\section{Experiments}

To evaluate the performance improvements of RTC-instrumented programs as a result of the static analyses described in this paper, we instrumented some programs from the SPECINT 95, SPECINT 2000, and Olden benchmark suites, as well as some of the programs used to evaluate Cyclone [10]. The programs were compiled with gcc, and executed on a $333 \mathrm{MHz}$ Pentium II running Linux. 
Table I. Benchmark Characteristics and Performance

\begin{tabular}{|c|c|c|c|c|c|c|c|}
\hline \multirow[b]{2}{*}{ Program } & \multirow{2}{*}{$\begin{array}{c}\text { Lines } \\
\text { of Code } \\
\text { (a) }\end{array}$} & \multicolumn{2}{|c|}{ Inst. RTC Routines } & \multirow{2}{*}{$\begin{array}{c}\text { Exec } \\
\text { time } \\
(\mathrm{d})\end{array}$} & \multirow{2}{*}{$\begin{array}{c}\text { RTC } \\
\text { slowd } \\
(\mathrm{e})\end{array}$} & \multirow{2}{*}{$\begin{array}{c}\text { opt } \\
\text { slowd } \\
\text { (f) }\end{array}$} & \multirow{2}{*}{$\begin{array}{c}\text { imprv } \\
\% \\
(\mathrm{~g})\end{array}$} \\
\hline & & $\begin{array}{l}\text { Static } \\
\text { (b) }\end{array}$ & $\begin{array}{c}\text { Dyn } \times 10^{6} \\
\text { (c) }\end{array}$ & & & & \\
\hline \multicolumn{8}{|l|}{ Cyclone } \\
\hline aes & 1822 & 2304 & 662.27 & 5.36 & 21.80 & 8.56 & 60.7 \\
\hline $\mathrm{cacm}$ & 340 & 182 & 330.46 & 4.01 & 27.01 & 6.87 & 74.6 \\
\hline cfrac & 4218 & 10092 & 1241.63 & 8.28 & 56.49 & 30.79 & 45.5 \\
\hline matxmult & 1377 & 109 & 463.33 & 4.76 & 6.50 & 3.45 & 46.9 \\
\hline ppm & 1421 & 1378 & 390.17 & 3.06 & 35.20 & 26.27 & 25.4 \\
\hline tile & 4880 & 4233 & 71.48 & 1.41 & 73.03 & 66.54 & 8.9 \\
\hline \multicolumn{8}{|l|}{ Olden } \\
\hline $\mathrm{bh}$ & 3200 & 1467 & 954.20 & 11.64 & 80.26 & 64.63 & 19.5 \\
\hline bisort & 690 & 603 & 774.25 & 5.68 & 25.96 & 16.40 & 36.8 \\
\hline em3d & 538 & 358 & 1065.55 & 11.97 & 6.82 & 4.89 & 28.2 \\
\hline health & 706 & 573 & 184.49 & 5.62 & 7.25 & 5.77 & 20.4 \\
\hline mst & 610 & 503 & 597.33 & 8.38 & 42.43 & 22.91 & 46.0 \\
\hline perimeter & 472 & 474 & 120.65 & 1.30 & 41.85 & 18.46 & 55.9 \\
\hline power & 867 & 1179 & 693.67 & 11.68 & 22.61 & 7.79 & 65.5 \\
\hline treeadd & 375 & 151 & 170.39 & 1.84 & 23.83 & 14.07 & 40.9 \\
\hline \multicolumn{8}{|l|}{ SPEC95 } \\
\hline compress & 3900 & 3095 & 3860.26 & 31.45 & 32.93 & 17.15 & 47.9 \\
\hline go & 29629 & 48163 & 1773.64 & 13.81 & 53.36 & 16.02 & 70.0 \\
\hline li & 7630 & 9406 & 955.60 & 4.57 & 74.21 & 64.65 & 12.9 \\
\hline \multicolumn{8}{|l|}{ SPEC2000 } \\
\hline bzip2 & 4650 & 4807 & 1239.41 & 7.01 & 41.64 & 28.59 & 31.3 \\
\hline gzip & 8605 & 6686 & 962.19 & 6.12 & 54.24 & 25.76 & 52.5 \\
\hline $\mathrm{mcf}$ & 2412 & 2234 & 163.70 & 1.74 & 15.75 & 11.63 & 26.2 \\
\hline
\end{tabular}

Table I lists some characteristics of the benchmarks we used, and the runtime performance results. Column (a) gives the program size in lines of code. Columns (b) and (c) give the static and dynamic number of calls to RTC functions (the functions listed in Section 2.2) added by the RTC tool: the goal of the static analyses presented in this paper is to reduce these numbers.

Column (d) gives the execution time (in seconds) of the uninstrumented code. Columns (e) and (f) give the runtime slowdown factor of the RTC-instrumented programs, before and after optimizing with the 


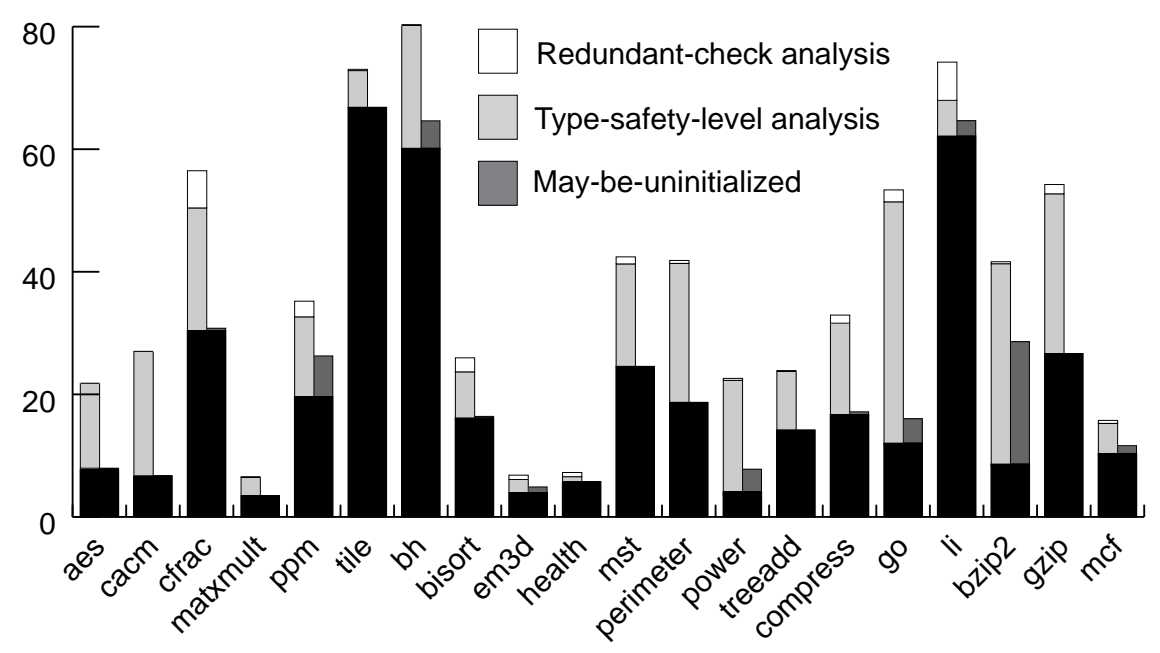

Figure \%. Execution time slowdown factor.

static analyses described in this paper; column (g) gives the percentage speedup gained from the optimization. On average, the unoptimized RTC-instrumented program (column (e)) ran 37 times slower than the uninstrumented executable, while the optimized version (column (f)) ran 23 times slower. On average, the optimizations improved execution time by $40.8 \%$ (column (g)).

Figure 7 graphs the slowdown factors, breaking down the effects of the various static analyses. The full height of the left-side bars represents the slowdown factor of the unoptimized RTC program (corresponding to Table I, column (e)). The two upper-left regions (white and light gray) represent the portion of runtime improvement due to the redundant-check and type-safety-level analyses respectively. The upper-right regions (dark gray) represent the slowdown due to instrumentation re-introduced by the may-be-uninitialized analysis. Thus, the height of the right-side bars (black plus dark gray) represents the slowdown factor of the optimized program (corresponding to column (f)). On average, the redundant-check analysis (white bar) improved performance by $3.6 \%$ (over the running time of the unoptimized RTC code); the type-safety-level analysis (light gray) improved performance by an additional $43.2 \%$; and the may-be-uninitialized analysis (dark gray) subtracted $6.0 \%$ from these improvements. 
Table II. Analysis Times (in seconds)

\begin{tabular}{lrrrr}
\hline & Normal Compl & Type-Safety & May-be-Uninit & Redund-Chk. \\
\hline cfrac & 6 & 0.41 & 0.71 & 5.52 \\
go & 19 & 2.84 & 166.14 & 8.87 \\
li & 10 & 2.79 & 304.74 & 133.57 \\
\hline
\end{tabular}

As an indicator of the efficiency of the different analyses, Table II gives the compilation and analysis times for the slower-compiling benchmarks; for the other benchmarks, all analyses took less than 1 second. Notice that the flow-insensitive type-safety-level analysis (the column labeled "type-safety"), which includes Das's points-to analysis, scales well, but the two flow-sensitive analyses are noticeably slower on larger programs. While some of this may be due to our suboptimal prototype implementation, the flow-sensitive analyses have a fundamentally higher complexity, so such behavior is expected.

Note also that if we allow the RTC tool to miss catching uses of uninitialized memory, we can skip the slow may-be-uninitialized analysis, and at the same time improve runtime performance (i.e., to the level of the black bars in Figure 7). The runtime overhead reintroduced by the may-be-uninitialized analysis is small overall but not insignificant. This is due in part to the fact that our implementation is intraprocedural; in particular, after a call to $f$, we conservatively consider everything in $\operatorname{MayMod}(\mathbf{f})$ as possibly uninitialized. An interprocedural implementation that builds a supergraph (which connects each call-site to the entry node of the called function), or incorporates context-sensitivity, is likely to be more precise, but will be slower.

\section{Future Work: Never-null-dereference Analysis}

One shortcoming of the type-safety-level analysis presented in Section 3 is that if a pointer $p$ is ever assigned a NULL value, then $* p$ is annotated as unsafe (and must thus be fully instrumented). This is because the

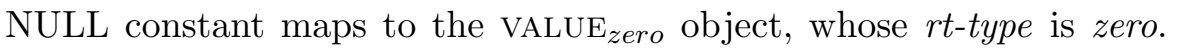


The assignment to $p$ causes $r$-type $(p)$ to be constrained to be no higher in the lattice than zero. This prevents $r$-type $(p)$ from being valid-ptr; thus, by Rule L2 in Figure 6, ${ }^{*} p$ is annotated as unsafe. This does not cause the RTC tool to miss any errors or to report any spurious errors, but it adds to the runtime overhead by including some unnecessary instrumentation.

With a small change to the type-safety-level analysis and another flow-sensitive analysis, we can find some instances of $* p$ where $p$ is guaranteed to contain either a valid pointer or a NULL value, and for each such instance, we can replace the verify-pointer instrumentation with a null-pointer check.

First, the rt-type lattice (in Figure 3 ) is modified so that the valid-ptr type is below the zero type, as follows:

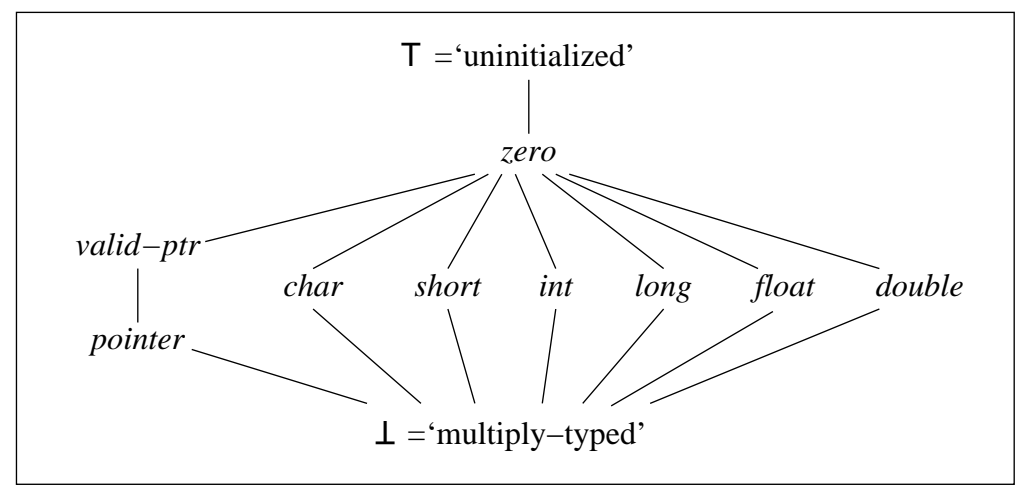

Second, Rule L2 in Figure 6 is changed to:

\begin{tabular}{|c|l|c|}
\hline & \multicolumn{1}{|c|}{ Condition } & Attribute \\
\hline \hline L2. & $\begin{array}{l}\text { static-type }(p)==\text { pointer, } \\
\text { rt-type }(p) \nsupseteq \text { valid-ptr }\end{array}$ & $* p$ unsafe \\
\hline
\end{tabular}

With these modifications, for any pointer $p$ that is only assigned valid pointer values or a NULL value, $* p$ will be safe. (Note that if a pointer may be assigned the result of pointer arithmetic or other invalid pointer values, then $* p$ will still be considered unsafe.)

Next, we perform another dataflow analysis to account for possible null-pointer dereferences of these pointers. This analysis is very similar to the may-be-uninitialized analysis described in Section 3.6. The 
lattice elements are sets of pointers, and the lattice meet is set union. The analysis computes the sets $\operatorname{Null}_{\text {in }}(n)$ and $N u l l_{\text {out }}(n)$ for each node $n$, representing the sets of pointers that may be null. The transfer functions for the different kinds of node $n$ are:

$-\quad p=\operatorname{NULL:} \operatorname{Null}_{\text {out }}(n)=\operatorname{Null}_{\text {in }}(n) \cup\{p\}$

$-\quad p=\& x: \operatorname{Null}_{\text {out }}(n)=\operatorname{Null}_{\text {in }}(n)-\{p\}$

$-p=q:$

if $q \in \operatorname{Null}_{\text {in }}(n)$, then $\operatorname{Null}_{\text {out }}(n)=\operatorname{Null}_{\text {in }}(n) \cup\{p\}$

else, $N_{u l l}$ out $(n)=\operatorname{Null}_{\text {in }}(n)-\{p\}$

$-p=* q$ :

if $p t-\operatorname{set}(q) \cap \operatorname{Null}_{\text {in }}(n) \neq \emptyset$, then $\operatorname{Null}_{\text {out }}(n)=\operatorname{Null}_{\text {in }}(n) \cup\{p\}$

else, $\operatorname{Null}_{\text {out }}(n)=\operatorname{Null}_{\text {in }}(n)-\{p\}$

$-\quad * p=q$ :

if $q \in \operatorname{Null}_{\text {in }}(n)$, then $\operatorname{Null}_{\text {out }}(n)=\operatorname{Null}_{\text {in }}(n) \cup p t$-set $(p)$

else, $\operatorname{Null}_{\text {out }}(n)=\operatorname{Null}_{\text {in }}(n)$

$-* p=* q:$

if $p t$-set $(q) \cap N u l l_{\text {in }}(n) \neq \emptyset$, then $\operatorname{Null}_{\text {out }}(n)=N u l l_{\text {in }}(n) \cup p t-\operatorname{set}(p)$

else, $\operatorname{Null}_{\text {out }}(n)=\operatorname{Null}_{\text {in }}(n)$

Upon completion of the analysis, a safe dereference $* p$ at node $n$ where $p \in \operatorname{Null}_{\text {in }}(n)$ must be instrumented to perform a null-pointer check.

\section{Related Work}

Many approaches have been proposed and developed that instrument a program to track auxiliary information during program execution.

Purify [8] is a commercial product that has proven to be successful in detecting buffer overruns, memory leaks, and other errors at runtime. It instruments object code, which gives it an advantage of not requiring source code, but a disadvantage of being platform dependent. Further, lack of source code means techniques such as that proposed 
in this paper cannot be applied to improve its overhead of about $15 \times$ slowdown.

Valgrind [17] is a similar tool, but it interprets the executable binary on a "synthetic CPU", and thus incurs a high overhead (about 40× slowdown). Its memcheck component mirrors each byte of memory with additional information, similar to the RTC tool. But since it operates on executable binaries, the techniques described in this paper cannot be directly applied to improve its performance.

Insure $++[15]$ is another heavyweight debugging tool that detects common sources of program errors, like out-of-bounds array accesses and null-pointer dereferences. Like the RTC tool, it instruments the program at the source level, so it is possible that using techniques similar to those presented in this paper could improve its performance.

Austin et al. [2] describe the Safe $C$ system, which tracks information about each pointer's referent, and uses this information to detect spatial (e.g., array out-of-bounds) and temporal (e.g., stale pointer dereference) access errors. They propose a compile-time optimization that is very similar to our redundant-check analysis. Patil et al. [16] describe a similar technique for checking spatial and temporal accesses called guarding, and propose a novel way to make this check more efficient by performing the tracking and checking of the auxiliary information in a shadow process on a separate processor.

Cyclone [10] and CCured [14] are two systems based on the C language that attempt to inject some level of safety while maintaining the low-level control of the language. The Cyclone language includes the definition of different kinds of pointers with different safety restrictions; "unsafe" pointer dereferences are instrumented with runtime checks (using "fat pointers" in a manner similar to $\mathrm{Safe}-\mathrm{C}$ ). To port an existing $\mathrm{C}$ program into Cyclone, the programmer must manually convert $\mathrm{C}$ pointers into the appropriate kind of Cyclone pointer to achieve optimal performance; an analysis to automatically classify pointers into the appropriate safety level, similar to the type-safety-level analysis proposed in this paper, would make it easier to port existing $\mathrm{C}$ code, and thus encourage greater use of this new language.

CCured also includes runtime checks for bad pointer dereferences. CCured's checks are more limited than RTC checks: specifically, CCured 
focuses only on pointers, and does not differentiate non-pointer types. Furthermore, CCured can be too strict (i.e., certain valid program behavior, such as storing the address of a stack variable in a global variable, or storing a pointer value in an integer, casting it back, and dereferencing it, will cause a runtime check to fail).

To reduce the overhead of runtime checks, CCured uses a typeinference scheme to identify as many safe and sequence pointers as possible, thus minimizing the amount of instrumented operations. The goal of their type inference is thus similar to that of our type-safety-level analysis. However, their type-inference scheme is less precise than our proposed analysis: they effectively group points-to sets into equivalence classes (in the spirit of Steensgaard's points-to analysis [20]), while our analysis accounts for the directionality of assignments. Despite this, they were able to significantly improve the performance of instrumented

CCured programs, from the unoptimized slowdown of 6-20 times, to between 1 and 2 times slowdown using the type-inference optimization.

The use of runtime checks to enforce safety properties, and techniques for eliminating unnecessary checks to improve performance, have been used in other programming languages and environments. Implementations of dynamically-typed languages like LISP and Scheme need to maintain runtime information to perform runtime type-checking as part of the language's semantics. To improve the performance of such a system, Henglein [9] proposes an efficient approach based on type inference. The Java language needs to perform potentially expensive runtime checks, such as array-bounds checks, to enforce safety properties guaranteed by the language. The elimination of redundant and unnecessary array-bounds checks in Java and other safe languages has been studied extensively $[21,13,7,3,4,12]$.

\section{Conclusions}

We have presented some techniques for reducing the runtime overhead of the instrumentation added to $\mathrm{C}$ programs by the Runtime Type-Checking tool. In Section 3, we defined a flow-insensitive typesafety-level analysis, which classifies each lvalue expression in the 
program as safe, unsafe, or tracked. In Section 4, we defined a dataflow analysis to identify redundant checks that can be eliminated. The results of these analyses, used in conjunction with the results of the may-be-uninitialized analysis defined in Section 3.6, improved the runtime performance of RTC by $40 \%$ on average, without sacrificing the ability of the RTC tool to detect errors.

Despite these improvements, the runtime overhead, averaging 23 times slower than the original program, remains relatively high. There is potential for developing other optimization techniques to improve performance. 


\section{References}

1. L. O. Andersen. Program Analysis and Specialization for the C Programming Language. PhD thesis, DIKU, University of Copenhagen, May 1994. (DIKU report 94/19).

2. T. Austin, S. Breach, and G. Sohi. Efficient detection of all pointer and array access errors. In ACM SIGPLAN Conference on Programming Language Design and Implementation (PLDI '94), SIGPLAN Notices 29(6), pages 290-201, Orlando, FL, June 1994.

3. R. Bodik, R. Gupta, and V. Sarkar. ABCD: Eliminating array bounds checks on demand. In ACM SIGPLAN Conference on Programming Language Design and Implementation (PLDI '00), SIGPLAN Notices 35(5), pages 321-333, Vancouver, BC, June 2000.

4. W.-N. Chin, S.-C. Khoo, and D. N. Xu. Deriving pre-conditions for array bound check elimination. In Proceedings of the Second Symposium on Programs as Data Objects, PADO 2001, pages 2-24, Aarhus, Denmark, May 2001.

5. Ckit. http://cm.bell-labs.com/cm/cs/what/smlnj/doc/ckit/.

6. M. Das. Unification-based pointer analysis with directional assignments. In ACM SIGPLAN Conference on Programming Language Design and Implementation (PLDI '00), SIGPLAN Notices 35(5), pages 35-46, Vancouver, BC, June 2000.

7. R. Gupta. Optimizing array bound checks using flow analysis. ACM Letters on Programming Languages and Systems, 2(1-4):135-150, March-December 1993.

8. R. Hasting and B. Joyce. Purify: Fast detection of memory leaks and access errors. In Proceedings of the Winter Usenix Conference, 1992.

9. F. Henglein. Global tagging optimization by type inference. In LISP and Functional Programming, pages 205-215, 1992.

10. T. Jim, G. Morrisett, D. Grossman, M. Hicks, J. Cheney, and Y. Wang. Cyclone: A safe dialect of C. In USENIX Annual Technical Conference, Monterey, CA, June 2002.

11. A. Loginov, S. Yong, S. Horwitz, and T. Reps. Debugging via run-time type checking. In Fundamental Approaches to Software Engineering (FASE), volume 2029 of Lec. Notes in Comp. Sci., pages 217-232. Springer, Apr. 2001.

12. M. Lujan, J. R. Gurd, T. L. Freeman, and J. Miguel. Elimination of java array bounds checks in the presence of indirection. Technical Report CSPP-13, Department of Computer Science, University of Manchester, Feb. 2002.

13. V. Markstein, J. Cocke, and P. Markstein. Optimization of range checking. In ACM SIGPLAN Symposium on Compiler Construction, SIGPLAN Notices 17(6), pages 114-119, Boston, MA, June 1982.

14. G. Necula, S. McPeak, and W. Weimer. CCured: Type-safe retrofitting of legacy code. In ACM Symposium on Principles of Programming Languages, Portland, OR, Jan. 2002. 
15. Parasoft. Insure++: An automatic runtime error detection tool, 2001. http://www.parasoft.com/insure/papers/tech.htm.

16. H. Patil and C. Fischer. Low-cost, concurrent checking of pointer and array accesses in C programs. Software-Practice and Experience, 27(27):87-110, 1997.

17. J. Seward. The design and implementation of Valgrind. Technical report, http://developer.kde.org/ ${ }^{\sim}$ sewardj/, 2000.

18. M. Siff, S. Chandra, T. Ball, K. Kunchithapadam, and T. Reps. Coping with type casts in C. In Proc. of ESEC/FSE '99: Seventh European Softw. Eng. Conf. and Seventh ACM SIGSOFT Symp. on the Found. of Softw. Eng., pages 180-198, Sept. 1999.

19. R. Stallman and R. Pesch. Using GDB: A Guide to the GNU Source-Level Debugger. July 1991.

20. B. Steensgaard. Points-to analysis in almost linear time. In ACM Symposium on Principles of Programming Languages, pages 32-41, 1996.

21. N. Suzuki and K. Ishihata. Implementation of an array bound checker. In ACM Symposium on Principles of Programming Languages, pages 132-143, Los Angeles, CA, Jan. 1977.

22. S. Yong, S. Horwitz, and T. Reps. Pointer analysis for programs with structures and casting. In ACM SIGPLAN Conference on Programming Language Design and Implementation (PLDI '90), SIGPLAN Notices 25(6), pages 91-103, Atlanta, GA, May 1999. 
rtc.tex; $15 / 10 / 2003 ; 17: 21 ;$ p.32 